\title{
Influence of the Local Scattering Environment on the Localization Precision of Single Particles
}

\author{
Dorian Bouchet $\odot,{ }^{1, *}$ Rémi Carminati $\odot,{ }^{2}$ and Allard P. Mosk $\oplus^{1}$ \\ ${ }^{1}$ Nanophotonics, Debye Institute for Nanomaterials Science, Utrecht University, P.O. Box 80000, 3508 TA Utrecht, Netherlands \\ ${ }^{2}$ Institut Langevin, ESPCI Paris, PSL University, CNRS, 1 rue Jussieu, 75005 Paris, France
}

(Received 30 August 2019; accepted 10 March 2020; published 1 April 2020; corrected 29 May 2020)

\begin{abstract}
We study the fundamental limit on the localization precision for a subwavelength scatterer embedded in a strongly scattering environment, using the external degrees of freedom provided by wavefront shaping. For a weakly scattering target, the localization precision improves with the value of the local density of states at the target position. For a strongly scattering target, the localization precision depends on the dressed polarizability that includes the backaction of the environment. This numerical study provides new insights for the control of the information content of scattered light by wavefront shaping, with potential applications in sensing, imaging, and nanoscale engineering.
\end{abstract}

DOI: 10.1103/PhysRevLett.124.133903

Localizing a small object in a complex scattering environment using wave scattering is a widespread problem in many fields, including material and life sciences. For instance, in nanofabrication, it is essential to control the manufacturing of structured samples and notably to localize defects in microelectromechanical systems (MEMS) [1], semiconductor chips [2,3], or photonic crystals [4]. In life sciences, studying the inner structure of the cell implies the localization of nanoparticles or fluorophores in scattering environments, for instance in particle tracking experiments $[5,6]$. Multiple scattering of acoustic waves or microwaves also complicates indoor localization of emitting or scattering devices $[7,8]$. Yet, for many applications, characterizing complex scattering materials by solving the inverse problem is still possible thanks to the large amount of prior information available to the observer through design considerations $[9,10]$. For this class of problems, defining and maximizing the information content of the data on a specific scattering object is a critical step in order to reach the best possible precision for imaging and metrology applications.

Estimation theory provides a definition of the precision in the estimation of a parameter (for example the position of a target) through the Cramér-Rao inequality [11]. This inequality sets a lower bound to the variance of the estimated value of the parameter, known as the CramérRao lower bound (CRLB). This bound depends on different features of the physical model, including the statistics of the measurement noise, the intrinsic properties of the scattering medium as well as the illumination and detection scheme. This theoretical limit has found useful applications in the design of optical imaging setups, for instance in the context of dynamic single-molecule measurements [12], diffuse optical imaging [13], or lifetime measurements [14,15]. The CRLB has also been proposed to define the resolution of an imaging system [16,17]. Furthermore, the concept is widely used to assess the localization precision in superresolution imaging techniques based on single-molecule detection [18-20]. Recently, the idea arose that the localization precision of single molecules could be improved by spatially modulating either the incident or the emitted field to minimize the CRLB [21,22]. In parallel, advanced wavefront protocols were developed to control wave propagation in strongly scattering media [23], notably enabling the focusing of light waves inside materials [24-29]. It is plausible that the localization precision for a hidden target can be improved by focusing light upon it, however this situation has not been rigorously analyzed so far.

In this Letter, we address this question by studying the fundamental limit in the precision on the localization of a subwavelength scatterer enclosed in a strongly scattering medium. We find out that the local environment of the target strongly influences the resulting localization precision. For a weakly scattering target (that is, when recurrent scattering between the target and the environment can be neglected), the key parameter driving the localization precision is the local density of states (LDOS), which is a fundamental quantity affecting many aspects of lightmatter interaction such as spontaneous emission and thermal emission [30,31]. For a strongly scattering target, the localization precision depends on the dressed polarizability of the scatterer, which describes the backaction of the environment beyond the weak-coupling regime [32]. These results offer new insights to improve the performances of imaging and metrology techniques using wavefront shaping.

We consider a model system composed of twodimensional scatterers arranged in a slab geometry, as represented in Fig. 1. One scatterer, located in the center of the system, is chosen as the target to be localized. The other 


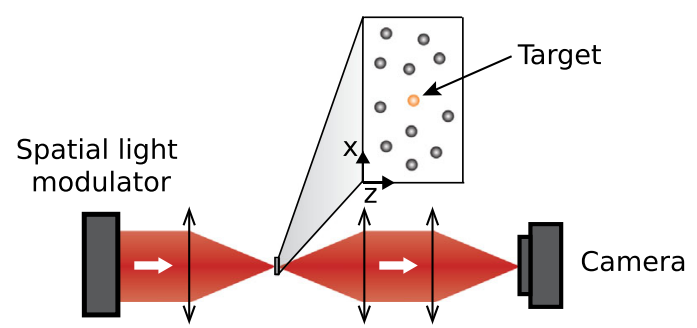

FIG. 1. Representation of a slab composed of several dipole scatterers and illuminated by a SLM. In all simulations, the thickness of the system is set to $L_{z}=10 \lambda$, and the transverse dimension is set to $L_{x}=50 \lambda$.

scatterers, with random positions, define a complex scattering medium. In this way, we can investigate universal properties of the localization precision of the target, without being influenced by features specific to a given scattering nanostructure. This model of a scattering medium has been used for the description of basic problems in mesoscopic physics [33,34], up to the regime of Anderson localization $[35,36]$. It is similar to that used in Ref. [37] to study the inverse reconstruction of the position of fluorophores. In order to constrain the problem, we assume that only the position $\mathbf{r}_{0}=\left(x_{0}, z_{0}\right)$ of the target is unknown. The goal is then to estimate the two coordinates of the target using coherent illumination at a wavelength $\lambda=2 \pi / k$, where $k$ is the wave number in vacuum. We further assume that the incident field is either a plane wave or a sum of plane waves with equal amplitude and different incidence angles, as generated in practice by a phase-only spatial light modulator (SLM). The response of the subwavelength scatterers is described by an electric polarizability $\alpha$ and a scattering cross section $\sigma_{s}=k^{3}|\alpha|^{2} / 4$. We denote the polarizability of the target by $\alpha_{0}$, and take its scattering cross section to be $\lambda / 1000$, ensuring that this scatterer is weakly coupled to its environment. We take the polarizability of the other scatterers at resonance $\left(\alpha=4 i / k^{2}\right)$, which is not an essential feature of the model but allows us to maximize their scattering cross sections, and therefore to minimize the number of scatterers needed to reach the multiplescattering regime. In order to compute the scattered field, we use the coupled dipole method, which is an exact formulation of the scattering problem in the limit of scatterers much smaller than the wavelength [38]. This method allows us to calculate the average (or expected) pixel intensity as measured by a camera located in the image plane of an ideal imaging system, which images the output plane $z=L_{z}$ (see Supplemental Material [39], Section I).

Any measurement process is intrinsically probabilistic due to noise fluctuations that limit the precision on the determination of the position of the target in otherwise perfect conditions. Thus, the measured data must be described by a random variable $\mathbf{X}$. The joint probability density function $p(\mathbf{X} ; \boldsymbol{\theta})$ of the dataset, parameterized by the set of unknown parameters $\boldsymbol{\theta}$ to be estimated, is used to define the Fisher information matrix [11]

$$
[\mathcal{F}(\boldsymbol{\theta})]_{j k}=\left\langle\left[\frac{\partial \ln p(\mathbf{X} ; \boldsymbol{\theta})}{\partial \theta_{j}}\right]\left[\frac{\partial \ln p(\mathbf{X} ; \boldsymbol{\theta})}{\partial \theta_{k}}\right]\right\rangle .
$$

Here $\langle\cdots\rangle$ denotes the average over noise fluctuations. While any noise statistics can be included in the formalism, we assume here that values measured on different pixels of the camera are statistically independent and follow a Poisson distribution, which corresponds to an experiment limited only by photon noise. The information matrix is then expressed by

$$
[\mathcal{F}(\boldsymbol{\theta})]_{j k}=\sum_{i=1}^{N} \frac{1}{I_{i}}\left(\frac{\partial I_{i}}{\partial \theta_{j}}\right)\left(\frac{\partial I_{i}}{\partial \theta_{k}}\right)
$$

where $N$ is the total number of pixels and $I_{i}$ is the average value of the intensity measured by the $i$ th pixel. From Eq. (2), we can compute the CRLB, which bounds the error in the determination of the parameter $\theta_{j}$, by

$$
\mathcal{C}_{j}=\sqrt{\left[\mathcal{F}^{-1}(\boldsymbol{\theta})\right]_{j j}}
$$

While there exists no general methodology to build an efficient estimation algorithm that reaches the CRLB, maximum likelihood estimation is the most popular approach to obtain practical estimators that are asymptotically efficient [11]. Moreover, it is possible to obtain an explicit expression of such an estimator, in the limit of small parameter variations and for a large number of detected photons (see Supplemental Material [39], Section II). This estimator may be used to estimate the position of the target even when the positions of the scatterers that constitute the scattering environment are known with some uncertainties (see Supplemental Material [39], Section III).

The CRLB can be evaluated in our model system by computing the average value of the intensity reaching the camera pixels using the coupled dipole method, and by evaluating the derivatives in Eq. (2) using a finite difference scheme. As only the coordinates of the target need to be estimated, we define $\mathcal{C}=\left(\mathcal{C}_{x}, \mathcal{C}_{z}\right)$ where $\mathcal{C}_{x}$ and $\mathcal{C}_{z}$ are the CRLB on each coordinate. For the calculations, we choose $\lambda=633 \mathrm{~nm}$ and an average incident intensity (integrated over the invariant $y$ coordinate) of $I_{0}=10^{4}$ photons per $\mu \mathrm{m}$. One can then easily deduce the CRLB for other values of $\lambda$ and $I_{0}$ by noting that the CRLB scales with $\lambda$ and with $I_{0}^{-1 / 2}$. In order to study the influence of multiple scattering on the precision in the estimation of the target position, we generate different random configurations of the medium that we illuminate with a plane wave at normal incidence, and we study the statistical distribution of the Cramer-Rao bound, with the statistics now performed with respect to disorder. Changing the density of 


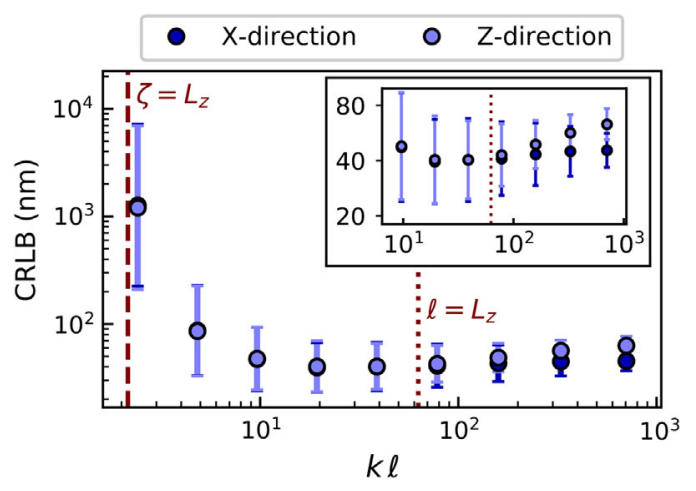

FIG. 2. CRLB as a function of the normalized scattering mean free path $k \ell$ for plane-wave illumination. Dotted lines correspond to $\ell=L_{z}$ (defining the transition to the multiple-scattering regime) and dashed lines correspond to $\zeta=L_{z}$ (defining the onset of Anderson localization). Each point represents the geometric mean over 1000 configurations of the disordered medium, and error bars represent 1-sigma intervals. The inset shows the same data on a smaller scale.

scatterers $\rho_{s}$ allows us to modify the independent scattering (or Boltzmann) mean free path $\ell=\left(\rho_{s} \sigma_{s}\right)^{-1}$ [40]. In Fig. 2 we show the first two moments of the CRLB distribution as a function of $k \ell$. In the single-scattering regime $\left(\ell \gtrsim L_{z}\right)$, the average CRLB depends on the coordinate to be estimated $\left(x_{0}\right.$ or $\left.z_{0}\right)$, as expected for one isolated scatterer. In contrast, for $\ell<L_{z}$, the average CRLB is the same for both coordinates due to multiple scattering that restores isotropy. In this regime, the probability distribution of the CRLB follows a log-normal distribution (see Supplemental Material [39], Section IV), whose moments strongly depend on the scattering mean free path. We also observe that the average CRLB shows a minimum in this regime, demonstrating that on average multiple scattering improves the localization precision. Finally, when the localization length becomes on the order of the size of the medium, the CRLB strongly increases due to the onset of Anderson localization which suppresses light transmission [41] [we use $\zeta=\ell \exp (\pi k \ell / 2)$ as a rough approximation of the localization length [42] ].

The CRLB provides a figure of merit that can be optimized using the external degrees of freedom provided by wavefront shaping. In order to test the optimization of information in the presence of multiple scattering, we generate 1000 configurations of the medium in the diffusive regime $\left(k \ell=9.7\right.$, optical thickness $L_{z} / \ell=6.5$ ), assumed to be illuminated using a phase-only SLM composed of $N_{e}=64$ elements. We then minimize the CRLB using a global optimization algorithm based on simulated annealing [43]. The optimized field distribution weakly depends on the initial guess fed to the optimization algorithm (see Supplemental Material [39], Section V), which suggests that the obtained solutions are close to the global optimum. We show in Fig. 3(a) and 3(b) the intensity
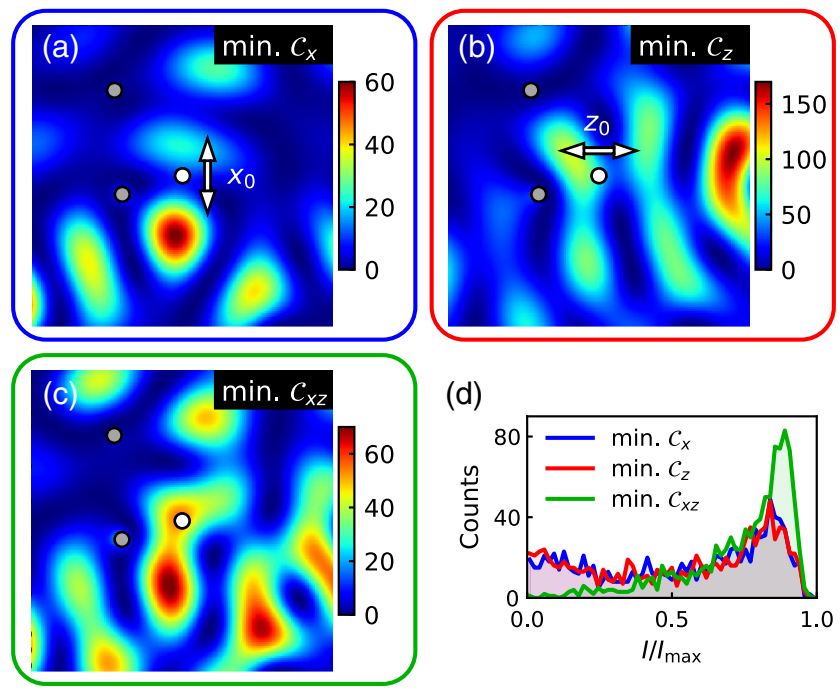

(d)

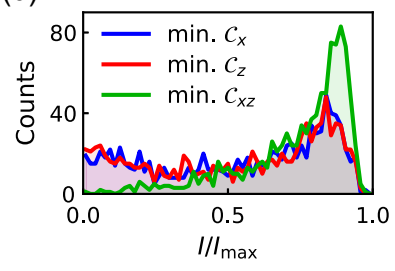

FIG. 3. Intensity $I / I_{0}$ around the target for a scattering medium illuminated by a wavefront optimized for (a) the transverse coordinate $x_{0}$, (b) the longitudinal coordinate $z_{0}$, and (c) both coordinates simultaneously. An area of $2 \lambda \times 2 \lambda$ is displayed on each map. (d) Histogram of the intensity ratio $I / I_{\max }$ at the target position when optimizing for $x_{0}, z_{0}$ and both coordinates simultaneously.

around the target for a scattering medium illuminated by incident fields independently optimized for the determination of $x_{0}$ and $z_{0}$, respectively. The incident wavefront associated with the highest information content depends on the coordinate to be estimated, with the appearance of intensity hot spots in the vicinity of the target. The formation of such hot spots might be interpreted as a possible trade-off between optimization of the intensity and of the intensity gradient at the target position. Comparing the intensity $I$ at the target position when optimizing the CRLB to the intensity $I_{\max }$ obtained after a direct optimization of the intensity on the target, we observe that the intensity ratio $I / I_{\max }$ varies from zero to one [Fig. 3(d)]. This confirms that determining the most informative wavefront cannot be reduced to a simple optimization of the intensity at the position of the targeted scatterer. We also show in Fig. 3(c) the intensity around the target obtained by minimizing a single CRLB associated with the estimation of both coordinates $\mathcal{C}_{x z}=\|\mathcal{C}\|_{2}$. In that case, we observe that optimizing the CRLB is not strictly equivalent to optimizing the intensity at the target position, but the distribution of $I / I_{\max }$ at the target position becomes strongly skewed towards unity [Fig. 3(d)].

In the shot-noise limit, the CRLB scales with the reciprocal of the square root of the number of interactions between the probe field and the target [44], which is here given by the power scattered by the dipole. Since this power is proportional to $|d|^{2}$ (where $d$ is the dipole induced in the target), on average, we can expect the CRLB to scale with $|d|^{-1}$. The induced dipole can be expressed as the sum of an 


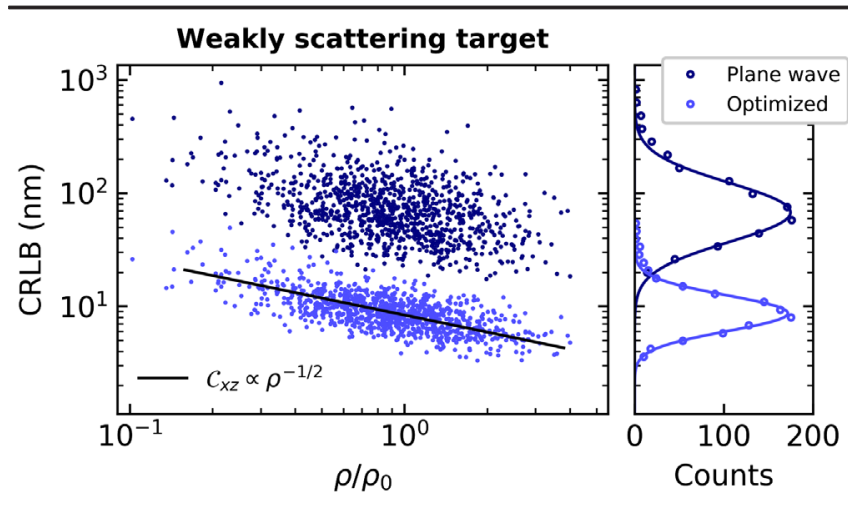

FIG. 4. Left: $\mathcal{C}_{x z}$ for a weakly scattering target as a function of the normalized LDOS. The black line is a fit to the optimized data by a power law with an exponent of $-1 / 2$ (a linear regression gives an exponent of -0.46). Right: Observed distribution of the CRLB. Log-normal distributions (solid lines) are fitted to numerical observations (data points).

excitation by the external local field, and a contribution resulting from backaction by the environment. This can be written as [32]

$$
d=\alpha_{0} \epsilon_{0} E_{\mathrm{exc}}\left(\mathbf{r}_{0}\right)+\alpha_{0} k^{2} S\left(\mathbf{r}_{0}, \mathbf{r}_{0}\right) d,
$$

where $S=G-G_{0}$ is the difference between the Green function in the presence of the medium and the free-space Green function, and $E_{\text {exc }}\left(\mathbf{r}_{0}\right)$ is the excitation field at the target position, generated by scattering of the incident field by the other scatterers. From Eq. (4), we can define a dressed polarizability $\tilde{\alpha}=\alpha_{0}\left[1-\alpha_{0} k^{2} S\left(\mathbf{r}_{0}, \mathbf{r}_{0}\right)\right]^{-1}$ such that $d=\tilde{\alpha} \epsilon_{0} E_{\text {exc }}\left(\mathbf{r}_{0}\right)$. This simple relation is a convenient starting point to investigate the cases of weakly and strongly scattering targets.

For a weakly scattering target $\left(\left|\alpha_{0} k^{2} S\left(\mathbf{r}_{0}, \mathbf{r}_{0}\right)\right| \ll 1\right)$, backaction from the medium is negligible and we can write $\tilde{\alpha} \approx \alpha_{0}$. In this regime, the strength of the induced dipole $|d|^{2}$ depends mainly on the intensity of the excitation field at the target position $\left|E_{\text {exc }}\left(\mathbf{r}_{0}\right)\right|^{2}$. After the optimization of $\mathcal{C}_{x z}$, we observe that the intensity at the target position scales with the LDOS (see Supplemental Material [39], Section VI). This is in agreement with a known result related to wave focusing in complex media by time reversal [45]. Consequently, we can expect the CRLB to scale with the reciprocal of the square root of the LDOS at the target position. To prove this assertion, we remove the target and calculate the LDOS $\rho=2 k /(\pi c) \operatorname{Im}\left[G\left(\mathbf{r}_{0}, \mathbf{r}_{0}\right)\right]$ at the target position. Introducing the free-space LDOS $\rho_{0}$, the normalized LDOS at the target position is then expressed by $\rho / \rho_{0}=1+4 \operatorname{Im}\left[S\left(\mathbf{r}_{0}, \mathbf{r}_{0}\right)\right]$. The normalized LDOS can be calculated numerically with the coupled dipole method, using a dipole source located at $\mathbf{r}_{0}$. Calculating $\rho / \rho_{0}$ and $\mathcal{C}_{x z}$ for 1000 configurations, we observe a negative correlation between the LDOS at the target position and the optimized CRLB (Fig. 4),

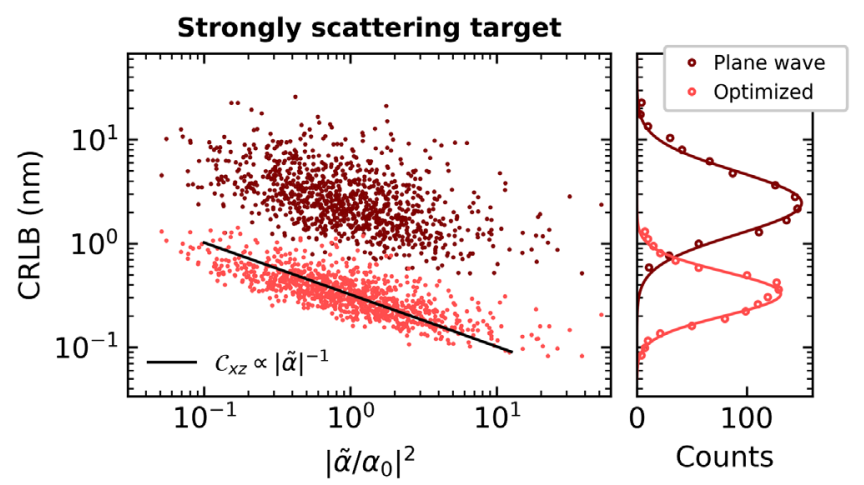

FIG. 5. Left: $\mathcal{C}_{x z}$ for a strongly scattering target as a function of the normalized effective scattering strength $\left|\tilde{\alpha} / \alpha_{0}\right|^{2}$. The black line is a fit to the optimized data by a power law with an exponent of $-1 / 2$ (a linear regression gives an exponent of -0.36 ). Right: Observed distribution of the CRLB. Log-normal distributions (solid lines) are fitted to numerical observations (data points).

characterized by a correlation coefficient of -0.69 calculated on log-transformed variables. This result clearly demonstrates that the localization precision of a weak scatterer improves with the value of the LDOS at its position. Furthermore, fitting a power law to numerical observations shows that the optimized CRLB scales with $\rho^{-1 / 2}$, which is the expected scaling of error in the shotnoise limit. We surmise that an even stronger correlation between $\rho / \rho_{0}$ and $\mathcal{C}_{x z}$ could be observed with a more complete control of input and output modes.

For a strongly scattering target that recurrently scatters the field, the interaction between the target and its environment has to be treated beyond the weak-coupling approximation. To investigate this regime, we set the polarizability of the target on resonance $\left(\alpha_{0}=4 i / k^{2}\right)$. The induced dipole depends on the dressed polarizability $\tilde{\alpha}$, which exhibits a pole for $\alpha_{0} k^{2} S\left(\mathbf{r}_{0}, \mathbf{r}_{0}\right)=1$. Near this pole, we can expect the strength of the induced dipole $|d|^{2}$ to scale with $|\tilde{\alpha}|^{2}$ and the CRLB to scale with $|\tilde{\alpha}|^{-1}$. This is confirmed by calculating $\left|\tilde{\alpha} / \alpha_{0}\right|^{2}$ and $\mathcal{C}_{x z}$ for 1000 configurations, as shown in Fig. 5. Indeed, we observe that $\left|\tilde{\alpha} / \alpha_{0}\right|^{2}$ and $\mathcal{C}_{x z}$ are strongly correlated (with a correlation coefficient of -0.77 calculated on logtransformed variables), and that the optimized CRLB roughly scales with $|\tilde{\alpha}|^{-1}$.

In summary, we have introduced a rigorous framework to study and optimize the precision of localization measurements for a subwavelength scatterer in a complex medium. For a weakly scattering target, we have shown that the lower bound on the localization precision depends on the LDOS at the target position. In contrast, the localization precision is driven by the dressed polarizability when recurrent scattering is significant. These results clarify the role of multiple scattering effects for metrology and imaging applications, as well as resulting fundamental limitations [46]. Our work also 
opens interesting perspectives in different research areas, for instance to track biological nanoparticles in complex environments [6] or to precisely estimate critical parameters of nanomanufactured samples [3]. While we have shown that the CRLB can be calculated and optimized using electromagnetic simulations, optimal wavefronts could also be experimentally identified in unknown scattering media by physically modulating the position of the target, for instance using ultrasound-based techniques $[47,48]$. Finally, we emphasize that the results are not limited to light waves, and apply to all kinds of waves, for instance to assess and optimize the localization precision of acoustic sources [49] or in microwave scattering experiments [50].

The authors thank S. Faez, D. van Oosten, and S. Skipetrov for insightful discussions and C. de Kok for IT support. This work was supported by the Netherlands Organization for Scientific Research NWO (Vici 68047618 and Perspective P16-08) and by LABEX WIFI (Laboratory of Excellence ANR-10-LABX-24) within the French Program "Investments for the Future" under reference ANR-10-IDEX-0001-02 PSL*

d.f.bouchet@uu.nl

[1] W. Osten, Optical Inspection of Microsystems (CRC Press, Boca Raton, 2019).

[2] E. Vogel, Nat. Nanotechnol. 2, 25 (2007).

[3] J. Alexander Liddle and G. M. Gallatin, Nanoscale 3, 2679 (2011).

[4] A. F. Koenderink, A. Lagendijk, and W. L. Vos, Phys. Rev. B 72, 153102 (2005).

[5] P. Zijlstra and M. Orrit, Rep. Prog. Phys. 74, 106401 (2011).

[6] R. W. Taylor and V. Sandoghdar, Nano Lett. 19, 4827 (2019).

[7] G. Deak, K. Curran, and J. Condell, Comput. Commun. 35, 1939 (2012).

[8] J. Xiao, Z. Zhou, Y. Yi, and L. M. Ni, ACM Comput. Surv. 49, 25 (2016).

[9] A. Szameit, Y. Shechtman, E. Osherovich, E. Bullkich, P. Sidorenko, H. Dana, S. Steiner, E. B. Kley, S. Gazit, T. Cohen-Hyams et al., Nat. Mater. 11, 455 (2012).

[10] T. Zhang, C. Godavarthi, P. C. Chaumet, G. Maire, H. Giovannini, A. Talneau, M. Allain, K. Belkebir, and A. Sentenac, Optica 3, 609 (2016).

[11] S. Kay, Fundamentals of Statistical Processing, Volume I: Estimation Theory (Prentice Hall, Upper Saddle River, 1993).

[12] L. P. Watkins and H. Yang, Biophys. J. 86, 4015 (2004).

[13] M. Boffety, M. Allain, A. Sentenac, M. Massonneau, and R. Carminati, Opt. Lett. 33, 2290 (2008).

[14] M. Köllner and J. Wolfrum, Chem. Phys. Lett. 200, 199 (1992).

[15] D. Bouchet, V. Krachmalnicoff, and I. Izeddin, Opt. Express 27, 21239 (2019).
[16] S. Ram, E. S. Ward, and R. J. Ober, Proc. Natl. Acad. Sci. U.S.A. 103, 4457 (2006).

[17] A. Sentenac, C.-A. Guérin, P. C. Chaumet, F. Drsek, H. Giovannini, N. Bertaux, and M. Holschneider, Opt. Express 15, 1340 (2007).

[18] R. J. Ober, S. Ram, and E. S. Ward, Biophys. J. 86, 1185 (2004).

[19] K. I. Mortensen, L. S. Churchman, J. A. Spudich, and H. Flyvbjerg, Nat. Methods 7, 377 (2010).

[20] H. Deschout, F. C. Zanacchi, M. Mlodzianoski, A. Diaspro, J. Bewersdorf, S. T. Hess, and K. Braeckmans, Nat. Methods 11, 253 (2014).

[21] Y. Shechtman, S. J. Sahl, A. S. Backer, and W. E. Moerner, Phys. Rev. Lett. 113, 133902 (2014).

[22] F. Balzarotti, Y. Eilers, K. C. Gwosch, A. H. Gynnå, V. Westphal, F. D. Stefani, J. Elf, and S. W. Hell, Science 355, 606 (2017).

[23] A. P. Mosk, A. Lagendijk, G. Lerosey, and M. Fink, Nat. Photonics 6, 283 (2012).

[24] I. M. Vellekoop, E. G. v. Putten, A. Lagendijk, and A. P. Mosk, Opt. Express 16, 67 (2008).

[25] C.-L. Hsieh, Y. Pu, R. Grange, and D. Psaltis, Opt. Express 18, 12283 (2010).

[26] Y. Choi, T. R. Hillman, W. Choi, N. Lue, R. R. Dasari, P. T. C. So, W. Choi, and Z. Yaqoob, Phys. Rev. Lett. 111, 243901 (2013).

[27] C. Ma, X. Xu, Y. Liu, and L. V. Wang, Nat. Photonics 8, 931 (2014).

[28] P. Ambichl, A. Brandstötter, J. Böhm, M. Kühmayer, U. Kuhl, and S. Rotter, Phys. Rev. Lett. 119, 033903 (2017).

[29] H. Ruan, T. Haber, Y. Liu, J. Brake, J. Kim, J. M. Berlin, and C. Yang, Optica 4, 1337 (2017).

[30] W. L. Barnes, J. Mod. Opt. 45, 661 (1998).

[31] R. Carminati, A. Cazé, D. Cao, F. Peragut, V. Krachmalnicoff, R. Pierrat, and Y. De Wilde, Surf. Sci. Rep. 70, 1 (2015).

[32] D. Bouchet and R. Carminati, J. Opt. Soc. Am. A 36, 186 (2019).

[33] P. de Vries, D. V. van Coevorden, and A. Lagendijk, Rev. Mod. Phys. 70, 447 (1998).

[34] S. Rotter and S. Gigan, Rev. Mod. Phys. 89, 015005 (2017).

[35] A. Cazé, R. Pierrat, and R. Carminati, Phys. Rev. Lett. 111, 053901 (2013).

[36] S. E. Skipetrov and I. M. Sokolov, Phys. Rev. Lett. 112, 023905 (2014).

[37] N. Irishina, M. Moscoso, and R. Carminati, Opt. Lett. 37, 951 (2012).

[38] M. Lax, Phys. Rev. 85, 621 (1952).

[39] See Supplemental Material at http://link.aps.org/ supplemental/10.1103/PhysRevLett.124.133903 for details about numerical methods and additional numerical results.

[40] A. Lagendijk and B. A. van Tiggelen, Phys. Rep. 270, 143 (1996).

[41] D. Vollhardt and P. Wölfle, Phys. Rev. B 22, 4666 (1980).

[42] B. C. Gupta and Z. Ye, Phys. Rev. E 67, 036606 (2003).

[43] S. Kirkpatrick, C. D. Gelatt, and M. P. Vecchi, Science 220, 671 (1983). 
[44] V. Giovannetti, S. Lloyd, and L. Maccone, Nat. Photonics 5, 222 (2011).

[45] R. Carminati, R. Pierrat, J. de Rosny, and M. Fink, Opt. Lett. 32, 3107 (2007).

[46] J. Girard, G. Maire, H. Giovannini, A. Talneau, K. Belkebir, P. C. Chaumet, and A. Sentenac, Phys. Rev. A 82, 061801(R) (2010).

[47] X. Xu, H. Liu, and L. V. Wang, Nat. Photonics 5, 154 (2011).
[48] B. Judkewitz, Y. M. Wang, R. Horstmeyer, A. Mathy, and C. Yang, Nat. Photonics 7, 300 (2013).

[49] T. Kundu, Ultrasonics 54, 25 (2014).

[50] P. del Hougne, M. F. Imani, M. Fink, D. R. Smith, and G. Lerosey, Phys. Rev. Lett. 121, 063901 (2018).

Correction: A missing radical sign has been inserted in Eq. (3). 